\title{
Akut dekompensierte Herzinsuffizienz - Salz- und Trinkmengenrestriktion ohne messbaren Nutzen
}

\author{
Markun, Stefan
}

DOI: https://doi.org/10.1024/1661-8157/a001448

Other titles: Acute congestive heart failure - salt and drinking quantity restriction without measureable benefit

Posted at the Zurich Open Repository and Archive, University of Zurich ZORA URL: https://doi.org/10.5167/uzh-90675

Journal Article

Accepted Version

Originally published at:

Markun, Stefan (2013). Akut dekompensierte Herzinsuffizienz - Salz- und Trinkmengenrestriktion ohne messbaren Nutzen. Praxis, 102(21):1325-1326.

DOI: https://doi.org/10.1024/1661-8157/a001448 


\title{
Akut dekompensierte Herzinsuffizienz - Salz--und \\ Trinkmengenrestriktion ohne messbaren Benefit
}

\author{
Frage: \\ Hat eine aggressive Salz- und Trinkmengenrestriktion einen Einfluss auf Gewicht und klinische \\ Stabilisierung bei hospitalisierten Patienten mit akut dekompensierter Herzinsuffizienz? \\ Hintergrund: \\ Ein Eckpfeiler der Therapie bei akuter Herzinsuffizienz ist die Wiederherstellung der meist \\ gestörten Euvolämie. Nebst den primär verwendeten Medikamenten werden zu diesem Zweck \\ auch Salz und Trinkmengenrestriktion empfohlen. Hier ist allerdings eine Kontroverse \\ entstanden, da sich Untersuchungen häufen, die keinen positiven Effekt dieser Behandlungen \\ finden konnten. Diese Studie überprüft bei hospitalisierten Patienten mit akut \\ dekompensierter Herzinsuffizienz, ob eine aggressive Natrium- und Trinkmengenrestriktion \\ einen Effekt auf den Gewichtsverlust oder auf die klinische Stabilisierung hat.
}

\section{Einschlusskriterien:}

- Hospitalisierte Patienten mit akut dekompensierter Herzinsuffizienz (LVEF 45\% oder geringer, Boston Kriterien $>7$ Punkte)

- Seit höchstens 36 Stunden hospitalisiert

\section{Ausschlusskriterien:}

- Kreatininclearance $<30 \mathrm{ml} / \mathrm{min}$, kardiogener Schock, Einschränkung der Lebenserwartung aufgrund Nebendiagnosen

- Zustände, die die Einhaltung der Therapie erschweren (z.B. Demenz)

\section{Studiendesign und Methode:}

Randomisierte Studie, Randomisierung innerhalb von 36 Stunden nach Spitaleintritt, Verblindung der medizinischen Betreuungspersonen

Studienort:

Öffentliches Lehrkrankenhaus, Porto Alegre, Brasilien

\section{Interventionen:}

- Interventionsgruppe: Trinkmengenbeschränkung auf maximal $800 \mathrm{ml}$ pro Tag, Kochsalzeinschränkung auf maximal $800 \mathrm{mg}$ Natrium pro Tag für sieben Tage, oder bis zum Spitalaustritt

- Kontrollgruppe: Normale Spitalkost, liberal bezüglich Flüssigkeit und Natrium

\section{Outcome:}

Primärer outcome

- Gewichtsverlust nach drei Tagen

- Klinische Stabilität nach drei Tagen definiert als Rückgang von Stauungszeichen gemäss einem Punktesystem und Absetzen der intravenösen Behandlung auf Herzinsuffizienz

Sekundäre outcomes

- Durstgefühl auf visueller Analogskala 0-10, Rehospitalisierung innerhalb von 30 Tagen aufgrund von Herzinsuffizienz 
- 75 Patienten wurden in die Studie eingeschlossen, im Mittel waren die Patienten 60 Jahre alt und zu 69\% Männer, die Baselinecharakteristika der Gruppen unterschieden sich nicht signifikant, insbesondere der mittlere Schweregrad der Herzinsuffizienz war vergleichbar.

- Der Gewichtsverlust innerhalb von drei Tagen, war in der Interventionsgruppe $4.4 \mathrm{~kg}$, in der Kontrollgruppe $4.7 \mathrm{~kg}$ (kein signifikanter Unterschied). Ebenfalls kein Unterschied fand sich bei der klinischen Stabilität, sowohl ausgedrückt durch das Punktesystem basierend auf Stauungszeichen, als auch durch die Verschreibungen von intravenösen Medikamenten gegen Herzinsuffizienz. Der Wechsel von intravenös applizierten Schleifendiuretika zur oralen Form fand in beide Gruppen im Median nach vier Tagen statt.

- Die Patienten waren durchschnittlich 6 Tage hospitalisiert in der Interventionsgruppe, 7 Tage in der Kontrollgruppe (kein signifikanter Unterschied). Kein Unterschied fand sich ebenfalls bezüglich der Rehospitalisierungsrate innerhalb von 30 Tagen. Das protokollierte Durstgefühl war in der Interventionsgruppe nach drei Tagen signifikant stärker (im Mittel 5.1 versus 3.4 von maximal 10 Punkten). In der Kontrolluntersuchung nach 30 Tagen wiesen die Patienten in der Interventionsgruppe signifikant mehr klinische Stauungszeichen auf.

\section{Kommentar:}

- Die Studie testet die Salz und Trinkmengenrestriktion bei Patienten mit akut dekompensierter Herzinsuffizienz und stellt keine Auswirkungen dieser Behandlung auf den Gewichtsverlauf oder die klinische Stabilität fest innerhalb von drei Tagen.

- Die Studie definiert zwei primäre Outcomes. Der primäre Outcome der klinischen Stabilisierung ist schwer zu interpretieren, da er sich aus Kriterien zusammensetzt, die anfällig für Verzerrung sind: Die klinische Beurteilung der Stauungszeichen einerseits unterliegt der Einschätzung eines Assessors. Dieser war gemäss Protokoll zwar verblindet, allerdings ist unklar in wieweit die Gruppenzugehörigkeit trotzdem zu erahnen war z.B. anhand vom Trinkflaschen oder Gläser am Patientenbett, oder durch direkte Information ausgehend vom Patienten selbst. Das zweite Kriterium, das den primären Outcome "klinische Stabilisierung" definiert war das Absetzen der intravenösen Behandlung für Herzinsuffizienz. Ein Zusammenhang zwischen der Stabilität des Patienten und der pharmakologischen Therapie ist zwar anzunehmen, dieser ist allerdings nicht genauer beschrieben. Man könnte auch annehmen, dass Ärzte in dieser Klinik Ihre Verschreibungen generell eher vorsichtig umstellen, und Änderungen nach drei Tagen deshalb generell selten zu beobachten sind. Das gemessene Körpergewicht allerdings in ein objektiver Messwert und sehr stabiler primärer Outcome.

- Dass sich die Trinkmengenrestriktion in dieser Studie nicht auf das Körpergewicht ausgewirkt hat, erscheint überraschend und stellt etablierte Behandlungskonzepte infrage. Neben der pharmakologischen Behandlung scheint die Salz- und Trinkmengenrestriktion möglicherweise eine untergeordnete Rolle zu spielen. Ob sich diese Resultate als allgemeingültig herausstellen und in Anpassungen der Therapieempfehlungen resultieren wird sich zeigen.

\section{Literatur:}

Aliti GB, Rabelo ER, Clausell N, Rohde LE, Biolo A, Beck-da-Silva L. Aggressive fluid and sodium restriction in acute decompensated heart failure: a randomized clinical trial. JAMA Intern med. 2013;173(12):1058-1064

\section{Verfasser:}

Stefan Markun 